Discussiones Mathematicae

Graph Theory 41 (2021) 1041-1050

https://doi.org/10.7151/dmgt.2227

\title{
SUPERMAGIC GRAPHS WITH MANY DIFFERENT DEGREES
}

\author{
Petr Kovář ${ }^{1}$, Michal KravČenko ${ }^{1}$, Adam Silber ${ }^{1}$ \\ Department of Applied Mathematics and \\ The IT4Innovations National Supercomputing Center \\ VS̆B - Technical University of Ostrava \\ 70833 Ostrava-Poruba, Czech Republic \\ e-mail: petr.kovar@vsb.cz
}

AND

\section{MATĚJ KRBEČEK}

Department of Applied Mathematics

Vঙ̆B - Technical University of Ostrava

70833 Ostrava-Poruba, Czech Republic

\begin{abstract}
Let $G=(V, E)$ be a graph with $n$ vertices and $e$ edges. A supermagic labeling of $G$ is a bijection $f$ from the set of edges $E$ to a set of consecutive integers $\{a, a+1, \ldots, a+e-1\}$ such that for every vertex $v \in V$ the sum of labels of all adjacent edges equals the same constant $k$. This $k$ is called a magic constant of $f$, and $G$ is a supermagic graph.

The existence of supermagic labeling for certain classes of graphs has been the scope of many papers. For a comprehensive overview see Gallian's Dynamic survey of graph labeling in the Electronic Journal of Combinatorics. So far, regular or almost regular graphs have been studied. This is natural, since the same magic constant has to be achieved both at vertices of high degree as well as at vertices of low degree, while the labels are distinct consecutive integers.
\end{abstract}

Keywords: graph labeling, supermagic labeling, non-regular graphs.

2010 Mathematics Subject Classification: 05C70, 05 C78.

\footnotetext{
${ }^{1}$ This work was supported by The Ministry of Education, Youth and Sports from the National Programme of Sustainability (NPU II) project "IT4Innovations excellence in science - LQ1602".
} 


\section{Definitions and Known Results}

Let $G$ be a simple graph with vertex set $V$ and edge set $E$. We denote the number of vertices by $n$ and the number of edges by $e$.

Let $f$ be an injective mapping of $E$ to $\mathbb{N}$. The weight of a vertex $v$ from $V$ is the sum of labels of all edges incident to $v$ and is denoted by $w(v)$. Thus,

$$
w(v)=\sum_{\substack{u v \\ v \in u v}} f(u v) .
$$

If the weight of every vertex $v \in V$ is the same, then $f$ is a magic labeling. The concept of magic labeling was introduced by Sedláček in [13]. Moreover, if the set of labels in $f$ consists of consecutive integers $\{a, a+1, a+e-1\}$, then $f$ is a supermagic labeling and every graph that allows such labeling is a supermagic graph.

Supermagic graphs were first introduced by Stewart. In [16] it is shown that the classic concept of an $n \times n$ magic square corresponds to a supermagic labeling of $K_{n, n}$. In [17] Stewart characterized supermagic complete graphs.

Sedláček [12] showed that Möbius ladders $M_{n}$ are supermagic when $n \geq 3$ and $n$ is odd, and that $C_{n} \times P_{2}$ is not supermagic when $n \geq 4$ and $n$ is even. Bača, Holländer, and Lih described two special classes of supermagic quartic graphs [1].

In [15] it is shown that if $G$ is an $r$-regular supermagic graph, then so is the composition of $G$ and $K_{n}$ for $n \geq 3$. The complete $m$-partite graph $K_{n, n, \ldots, n}$ is supermagic when $n \geq 3, m>5$ and $m \neq \equiv 0(\bmod 4)$, and also the composition of $C_{m}$ and $K_{n}$ is supermagic if $m \geq 3$ and $n \geq 2$. Ho and Lee [4] showed that the graph $K_{m}\left[\bar{K}_{n}\right]$ is supermagic if $m=3$ or $m=5$ and $n=2$ or $n$ is odd. Shiu, Lam, and Cheng [14] proved that $m K_{n, n}$ is supermagic for $n \geq 2$ if and only if $n$ is even or both $m$ and $n$ are odd. In [7] Ivančo characterized supermagic regular complete multipartite graphs and supermagic cubes. He showed that $Q_{n}$ is supermagic if and only if $n=1$ or $n$ is even and $n>2$. He also proved that $C_{n} \times C_{n}$ and $C_{2 m} \times C_{2 n}$ are supermagic and conjectured that $C_{m} \times C_{n}$ is supermagic for all $m$ and $n$. In [2] some lower and upper bounds for numbers of edges in supermagic graphs are established. Ivančo, Lastivková, and Semaničová [8] characterized magic line graphs of general graphs and supermagic line graphs of regular bipartite graphs.

Hartsfield and Ringel in [5] presented a simple construction of non-regular supermagic graphs. They proved that in an $r$-regular supermagic graph there exists an edge $e$ such that $G-e$ is supermagic. In [9] Ivančo and Polláková proved that complete multipartite graphs $K_{1, n, n}$ are supermagic if and only if $n \geq 2$ and $K_{1,2, \ldots, 2}$ is supermagic.

Some examples of infinite classes of non-regular supermagic graphs have already been presented by Hartsfield, Ringel, and Stewart in [6] and [16]. Further 
examples have been provided by Drajnová, Ivančo, and Semaničová in [2], and Ivančo and Semaničová in [10] and [11]. All the non-regular supermagic graphs in papers $[2,5,6,10,11]$ and [16] have only two different degrees of vertices. A comprehensive overview on supermagic graphs is in [3].

Tomáš Madaras in a personal communication pointed out a very natural question: how irregular can a supermagic graph be? In this paper we try to answer that question. We give a construction of supermagic graphs with an arbitrarily high difference of degrees and another construction with arbitrarily many different degrees.

Let $G, H$ be two graphs. The composition $G[H]$, also called the lexicographic product of $G$ and $H$, is the graph with vertex set $V(G) \times V(H)$, and the edges between $\left(u_{1}, u_{2}\right)$ and $\left(v_{1}, v_{2}\right)$ of $G[H]$ are connected by an edge if $u_{1} v_{1} \in E(G)$ or if $u_{1}=v_{1}$ and $u_{2} v_{2} \in E(H)$. The results in Section 2 are based on compositions.

Let $G$ be a graph and let $H$ be a bipartite graph with both partite sets consisting of $r$ vertices. A bipartite composition $B C(G, H)$ is a graph that arises by replacing each vertex of $G$ with $r$ vertices and each edge of $G$ with a copy of $H$. The structure of $B C(G, H)$ is determined uniquely only if $H$ is vertex transitive. Otherwise, the structure of $B C(G, H)$ depends on which vertex of $H$ is mapped to which vertex in $B C(G, H)$. However, this ambiguity will be essential in constructing supermagic graphs with many different degrees. The results in Section 3 are based on bipartite compositions.

\section{Difference Between Maximal and Minimal Degrees}

Surprisingly, the difference between the highest and the lowest degree in a supermagic graph can be arbitrarily high. Here we provide a direct construction of such graphs along with the idea on which the construction is based. First we show that for odd $n$ the graph consisting of $t$ copies of $K_{n, n}$ allows an edge labeling such that the vertex weights in the $i$-th copy equal $i d$ for some fixed integer $d$.

Lemma 2.1. The graph $t K_{n, n}$, where $n$ is odd, allows an edge labeling by consecutive integers such that the vertex weight in the $i$-th copy of $K_{n, n}$ is $\mathrm{in}^{3}$.

Proof. In [16] it was shown that $K_{n, n}$ allows a supermagic labeling $f$ for any $n \geq 3$. Since $K_{n, n}$ is regular, without loss of generality, we assume that the smallest label in $f$ is 1 and thus the magic constant is $k=n\left(n^{2}+1\right) / 2$. Now adding $\left(n^{2}-1\right) / 2$ to each edge label we obtain a supermagic labeling $f_{1}$ of the first copy $K_{n, n}$ with the magic constant $k_{1}=n\left(n^{2}+1\right) / 2+n\left(n^{2}-1\right) / 2=n^{3}$.

Now assigning the next $n^{2}$ integers to the second copy of $K_{n, n}$ by adding yet another $n^{2}$ to each edge label we obtain a supermagic labeling $f_{2}$ of the second copy $K_{n, n}$ with the magic constant $k_{2}=n^{3}+n \cdot n^{2}=2 n^{3}$. Similarly, the $i$-th 
copy labeled by integers $i n^{2}+\left(1-n^{2}\right) / 2, i n^{2}+\left(1-n^{2}\right) / 2+1, \ldots, i n^{2}+\left(n^{2}-1\right) / 2$ has a supermagic labeling $f_{i}$ with the magic constant $k_{i}=i n^{3}$.

Clearly, all the $t n^{2}$ labels used in labeling $t K_{n, n}$ are consecutive integers starting at $\left(n^{2}-1\right) / 2+1$ and the vertex weight in the $i$-th copy of $K_{n, n}$ is $i n^{3}$. Thus, the union $f=\bigcup_{i=1}^{t} f_{i}$ is the desired labeling.

Consider the supermagic graph $G$ in Figure 1 on the left with magic constant $k=12$. It is isomorphic to $K_{3,3}-u v$. When multiplying each label by $d$, the vertex weight in the resulting graph is $12 d$ (Figure 1 on the right).
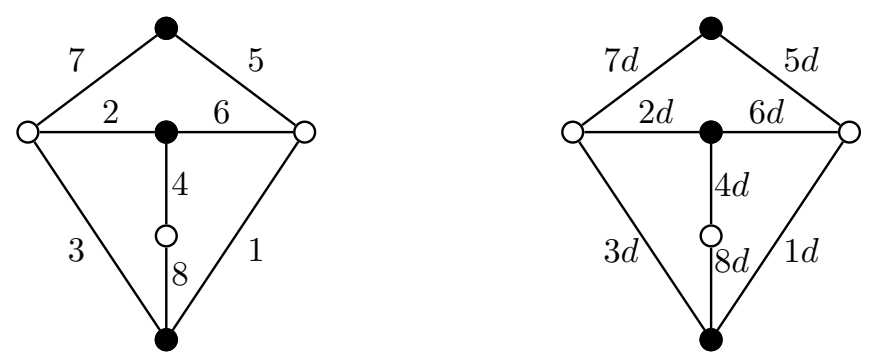

Figure 1. Supermagic labeling of $G$ and $G$ with labels multiplied by $d$.

The composition $G\left[\bar{K}_{n}\right]$ can be considered as replacing each edge labeled $i$ by the $i$-th copy of $K_{n, n}$ with vertex weight $i n^{3}$. Taking 8 copies of $K_{n, n}$ as in Lemma 2.1 we construct the composition $G\left[\bar{K}_{n}\right]$. Each edge labeled $i$ is replaced by $K_{n, n}$ with the vertex weight $i n^{3}$ as in Lemma 2.1. The resulting graph is supermagic (Figure 2).

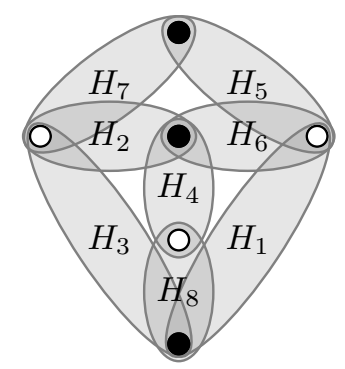

Figure 2. Supermagic labeling of $G\left[\bar{K}_{n}\right], G \simeq K_{3,3}-u v$.

Lemma 2.2. Let $G=K_{3,3}-u v$. The composition $G\left[\bar{K}_{n}\right]$ is supermagic for every odd integer $n$ and $\Delta\left(G\left[\bar{K}_{n}\right]\right)-\delta\left(G\left[\bar{K}_{n}\right]\right)=n$.

We omit the formal proof, since the claim is a special case of Theorem 2.3. 


\subsection{Generalization}

An analogous construction yields a supermagic labeling for any supermagic graph $G$. Previously, in [15] and [4] compositions $G[H]$ were considered for regular graphs $G$ only. The composition $G\left[\bar{K}_{n}\right]$ is supermagic also if $G$ is not regular, however $n$ must be odd.

Theorem 2.3. Let $G$ be any supermagic graph. The composition $G\left[\bar{K}_{n}\right]$ is supermagic for every odd integer $n$.

Proof. Let $G$ be any supermagic graph with $e$ edges and consecutive labels $a, a+1, \ldots, a+e-1$. In the composition $G\left[\bar{K}_{n}\right]$ replace each edge labeled $i$ with the $i$-th copy of $K_{n, n}$ and label the edges with the labeling $f$ from Lemma 2.1. The set of labels in $G$ consits of consecutive integers, so the set of labels in $G\left[\bar{K}_{n}\right]$ are consecutive integers $a n^{2}+\left(1-n^{2}\right) / 2, a n^{2}+\left(1-n^{2}\right) / 2+1, \ldots,(a+e-1) n^{2}+$ $\left(n^{2}-1\right) / 2$. The weight $k$ of every vertex of degree $d$ in $G$ is obtained as a sum of some $d$ integers $a_{1}, a_{2}, \ldots, a_{d}$. Let $f=\bigcup_{i=a}^{a+e-1} f_{i}$ be the labeling of edges in $G\left[\bar{K}_{n}\right]$. Then the weight of every vertex in $G\left[\bar{K}_{n}\right]$ is obtained as the sum of $n d$ integers: $n$ edges in the $a_{1}$-st copy of $K_{n, n}$ contribute the sum $a_{1} n^{3}$, the next $n$ edges in the $a_{2}$-nd copy contribute the sum $a_{2} n^{3}$ and so on. Thus, the weight is $a_{1} n^{3}+a_{2} n^{3}+\cdots+a_{d} n^{3}=k n^{3}$. Hence, $f$ is a supermagic labeling of $G\left[\bar{K}_{n}\right]$.

Now, the highest degree $\Delta\left(G\left[\bar{K}_{n}\right]\right)=3 n$ and the smallest degree $\delta\left(G\left[\bar{K}_{n}\right]\right)=$ $2 n$. Immediately we have the following.

Corollary 2.4. The difference between the highest and lowest degree in a supermagic graph can be arbitrarily high.

Remark 2.5. The same approach for even $n$ is not possible. The construction described in the proof of Theorem 2.3 requires the weights of copies of $K_{n, n}$ to form an arithmetic sequence with difference $n^{3}$ and first element $i n^{3}$, where $i$ is the smallest weight used in $G$. Hence, the first copy has to have vertex weights equal to $i n^{3}$, which can be achieved only by adding $\left((2 i-1) n^{2}-1\right) / 2$ to every label (due to Lemma 2.1). Yet, for even $n$, that is not an integer.

\section{Supermagic Graphs with Many Different Degrees}

Our main goal is to show that a supermagic graph can have arbitrarily many different degrees. First we construct graph $H-$ a building block that has the following properties: $H$ is bipartite with equal-sized partite sets, $H$ has at least two different degrees, and $t H$ is supermagic for any positive odd integer $t$. Then taking a suitable regular graph $G$, constructing a bipartite composition $B C(G, H)$ using a proper number of copies of $H$ (Figure 3 ) we obtain a larger supermagic 
graph $J$. The resulting graph $J$ can have several different degrees depending on the degrees of vertices shared by different copies of $H$ at each vertex of $J$.

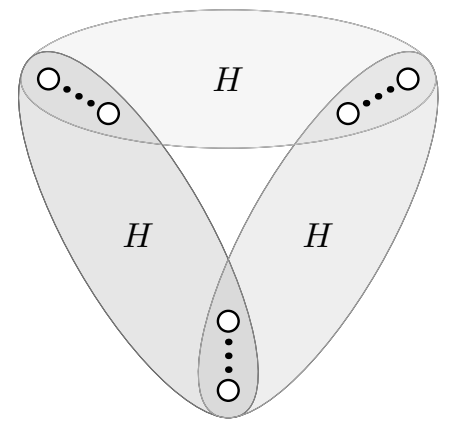

Figure 3. A bipartite composition $J$ obtained by gluing copies of $H$.

\subsection{The building block}

First we label edges of $t k 4$-cycles with consecutive integers $a, a+1, \ldots, a+4 t k-1$ as in Figure 4. To construct each building block $H$ we take a subset of any $k$ 4 -cycles among the $t k 4$-cycles. Notice, that the edge-labeling is a bijection. Each cycle has one vertex of small weight $2 a+2 t k-1$ (drawn in white), two vertices of medium weight $2 a+4 t k-1$ (gray) and one vertex of high weight $2 a+6 t k-1$ (black).

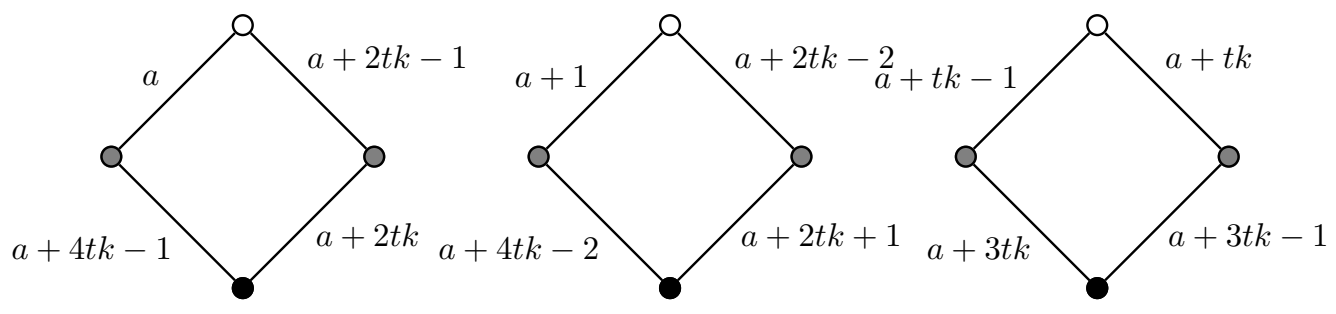

Figure 4. $k$ copies of $C_{4}$.

Suppose $p, q$, and $r$ are integers, $p+q=r$, and $p<q<r$. Now the supermagic bipartite graph $H$ is obtained by gluing together $k 4$-cycles as in Figure 5. Each 4-cycle will have the vertex with the smallest weight with respect to the cycle among $p$ vertices of high degree, two vertices with the middle weight among $r$ vertices of medium degree, and the vertex with the highest weight among $q$ vertices of small degree. The vertex degrees correspond to the number of 4 cycles glued together at each vertex. There are $x 4$-cycles meeting at every vertex 
in $P, z 4$-cycles at every vertex in $Q$, and $y 4$-cycles at every vertex in $R$. If $2 x$ are the degrees in $P, 2 z$ are the degrees in $Q$, and $2 y$ are the degrees in $R$, then comparing the number of 4-cycles and the number of edges in each set we have

$$
2 k=2 x p, \quad 4 k=2 y r, \quad 2 k=2 z q .
$$

The weight of every vertex of the resulting graph $H$ has to be the same integer $M$. This yields the following equalities for vertex weight:

$$
M=x(2 a+2 t k-1), \quad M=y(2 a+4 t k-1), \quad M=z(2 a+6 t k-1) .
$$

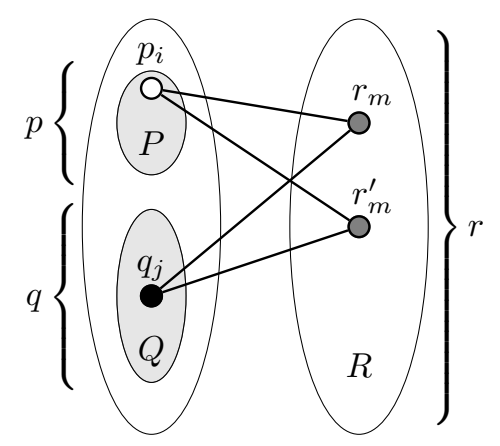

Figure 5. Structure of graph $H$.

Under the assumption that $H$ is a simple graph and $t=1$, the smallest values satisfying equations (1) and (2) we were able to find are $k=735, a=368$, $p=21, q=49, r=70, x=35, y=21, z=15$, and $M=77175$. Even though there are smaller integral solutions of the equations (1) and (2), the corresponding graph $H$ cannot have multiedges, therefore the number of edges leaving $P$ must satisfy $2 p x \geq r$. The inequality $2 q y \geq r$ is satisfied because $q \geq p$ and $2 r y \geq p+q$ holds because $p+q=r$. Notice that there are 735 different 4-cycles having one vertex in $P$, so each vertex in $P$ has $2 x=70$ neighbors, so the bipartite subgraph induced on $P \cup R$ is complete.

We choose the structure of $H$ as follows. We denote vertices in $P$ by $p_{i}$ for $i=0,1, \ldots, 20$, vertices in $Q$ by $q_{j}$ for $j=0,1, \ldots, 48$, and vertices in $R$ by $r_{m}$ and $r_{m}^{\prime}$ for $m=0,1, \ldots, 34$. Every pair of vertices $r_{m}, r_{m}^{\prime}$, for $m=0,1, \ldots, 34$ we join to all 21 vertices in $P$ and to 21 vertices $q_{j}$ in $Q$, where $j \equiv(21 m+i) \bmod 49$ for $i=0,1, \ldots, 20$ (21 consecutive subscripts modulo 49). Hereby we construct 21 4-cycles sharing $r_{m}$ and $r_{m}^{\prime}$ (see Figure 5). Altogether we construct $21 \cdot 35=735$ 4-cycles $r_{m} p_{i} r_{m}^{\prime} q_{j}$. These are edge-disjoint, since every edge between $P$ and $R$ appears in exactly one 4-cycle and no edge between $Q$ and $R$ is repeated, since every vertex $r_{m}$ (or $r_{m}^{\prime}$, respectively) is joined to 21 different vertices in $Q$. Clearly, the degrees of vertices in $P$ are $2 x=70$ and in $R$ are $2 y=42$. It 
is not difficult to see that the degrees of all vertices in $Q$ are $2 z=30$, since between $21 \cdot 35=735$ consecutive integers $j=21 m+i$, for $i=0,1, \ldots, 20$ and $m=0,1, \ldots, 34$, are 15 integers congruent modulo 49 , so each vertex in $Q$ is joined to 15 pairs of vertices in $R$.

We have the following lemma.

Lemma 3.1. Graph $H$ is supermagic, bipartite with equal-sized partite sets and three distinct degrees. Moreover, for every odd positive integer the graph $t H$ is supermagic.

Proof. Suppose $H$ is labeled as described above. We call the labeling $f$. Clearly, the resulting graph $H$ has $p+q=r=70$ vertices in each partite set. The labels are distinct consecutive integers starting at $a=368$. Graph $H$ is supermagic, because the weight of each vertex is $M=77175$, since $x(2 a+2 k-1)=y(2 a+4 k-1)=$ $z(2 a+6 k-1)=77175$. The vertices in $P$ are of degree $2 x=70$, the vertices in $Q$ are of degree $2 z=30$, and the vertices in $R$ are of degree $2 y=42$.

Finally, to show that $t$ copies of $H$ are also supermagic for positive odd $t$, we construct the following labeling $f^{\prime}$ of $t H$. Choosing $a=(735 t+1) / 2$ and $t k=735 t$ we label all $t k 4$-cycles as in Figure 4 with consecutive integers starting at $a$. Each 4 -cycle has the smallest weight $2 a+2 t k-1$, the medium weight $2 a+4 t k-1$, and the highest weight $2 a+6 t k-a$. Now, we glue every copy of $H$ from a different set of $k 4$-cycles as was shown above for $t=1$ and we obtain $t H$ in which each vertex has the weight $M=x(2 a+2 t k-1)=35(735 t+1+2 \cdot 735 t-1)=77175 t$. Similarly, $y(2 a+4 t k-1)=77175 t$ and $z(2 a+6 t k-1)=77175 t$. The resulting graph $t H$ is supermagic.

Remark 3.2. The graph $H$ described above was the smallest we were able to find. It has 140 vertices with different degrees in equal-sized partite sets $(70$ vertices of degree 42 in one partite set and 21 vertices of degree 70 along with 49 vertices of degree 30 in the second partite set). It has 2940 edges and therefore we provide no detailed figure of the graph.

If a smaller non-regular graph $H$ satisfying the above requirements is found, the construction in the following section will not be affected.

\subsection{Combining the blocks}

In the previous subsection we constructed a supermagic graph $t H$ with three different degrees $2 x>2 y>2 z$. Now we glue together copies of $H$ to construct a supermagic graph with arbitrarily many different degrees as in Figure 3.

Theorem 3.3. For every positive integer $k$ there exist supermagic graphs with at least $k$ different degrees. 
Proof. Let $G$ be an $s$-regular graph with $t$ edges, where $t$ is some odd integer. We denote the bipartite composition $B C(G, H)$ by $J$. The parameters $p, q, r, x$, $y$, and $z$ follow the notation in Lemma 3.1. Notice, that $J$ can be obtained by gluing together copies of $H$; at every vertex of $J$ always $s$ vertices from different copies of $H$ are glued together. Clearly, if $t H$ is a supermagic graph with magic constant $M$, then $J$ is supermagic as well with the magic constant $s M$. The degrees in $J$ can vary from $2 s z$ (obtained by gluing together $s$ vertices of the smallest degree $z$ ) to $2 s x$ (obtained by gluing together $s$ vertices of the highest degree $x$ ), depending on which vertices in the bipartite composition $J$ are glued together.

In particular, for every positive integer $k$ there exist supermagic graphs with $k$ different degrees. One can take a complete graph $K_{l}$, where $l \geq k$ and $l \equiv 2,3$ $(\bmod 4)$ and construct a suitable bipartite composition $B C\left(K_{l}, H\right)$. Again, we denote it by $J$. For such $l$ the number of edges in $K_{l}$ is odd, thus $J$ obtained by gluing together an odd number of copies of $H$ is always a supermagic graph. Every vertex $v_{i}$ of $K_{l}$ corresponds to $r$ vertices of $J$; by $V_{i}$ we denote the set of them. Each vertex in $V_{i}$ is shared by $l-1$ bipartite graphs $H$ and without loss of generality we construct $B C\left(K_{l}, H\right)$ so that one particular vertex in $V_{i}$ is of degree $2 x$ in $i$ copies of $H$ and of degree $2 z$ in the remaining $l-1-i$ copies of $H$. Hence, there are vertices of at least $l$ different degrees $2(l-1) x, 2(l-2) x+2 z$, $\ldots, 2(l-1) z$ in $J$. Since $l \geq k$, the claim follows.

Notice that the resulting graph is a bipartite composition, not a composition, since no $H$ is a complete bipartite graph.

\section{Conclusion}

We constructed an infinite class of supermagic graphs with an arbitrary difference between the highest and lowest degree, and an infinite class of graphs with arbitrarily many different degrees. Since the set of labels by definition has to consist of consecutive integers, such graphs are huge. However, there may exist smaller examples. Moreover, all the graphs constructed in Section 3 have all vertices of even degrees. This comes from convenience of the construction, yet we believe that supermagic graphs with many different degrees are not restricted to even degrees only. For example the graph $G$ in Figure 1 has both odd and even degrees, $K_{3,3}$ is supermagic and has only odd degrees. We post the following open problem.

Problem 4.1. Can a supermagic graph have arbitrarily many different odd degrees? 


\section{REFERENCES}

[1] M. Bača, I. Holländer and K.-W. Lih, Two classes of super-magic graphs, J. Combin. Math. Combin. Comput. 23 (1997) 113-120.

[2] S. Drajnová, J. Ivančo and A. Semaničová, Numbers of edges in supermagic graphs, J. Graph Theory 52 (2006) 15-26. https://doi.org/10.1002/jgt.20144

[3] J.A. Gallian, A dynamic survey of graph labeling, Electron. J. Combin. (2018) \#DS 6.

[4] Y.S. Ho and S.M. Lee, Some initial results on the supermagicness of regular complete $k$-partite graphs, J. Combin. Math. Combin. Comput. 39 (2001) 3-17.

[5] N. Hartsfield and G. Ringel, Supermagic and antimagic graphs, J. Recreat. Math. 21 (1989) 107-115.

[6] N. Hartsfield and G. Ringel, Pearls in Graph Theory: A Comprehensive Introduction (Academic Press, San Diego, 1990).

[7] J. Ivančo, On supermagic regular graphs, Math. Bohem. 125 (2000) 99-114. https://doi.org/10.21136/MB.2000.126259

[8] J. Ivančo, Z. Lastivková and A. Semaničová, On magic and supermagic line graphs, Math. Bohem. 129 (2004) 33-42.

https://doi.org/10.21136/MB.2004.134107

[9] J. Ivančo and T. Polláková, Supermagic graphs having a saturated vertex, Discuss. Math. Graph Theory 34 (2014) 75-84. https://doi.org/10.7151/dmgt.1711

[10] J. Ivančo and A. Semaničová, Some constructions of supermagic graphs using antimagic graphs, SUT J. Math. 42 (2006) 177-186.

[11] J. Ivančo and A. Semaničová, Some constructions of supermagic non-regular graphs, Australas. J. Combin. 38 (2007) 127-139.

[12] J. Sedláček, On magic graphs, Math. Slovaca 26 (1976) 329-335.

[13] J. Sedláček, Problem 27, in: Theory of Graphs and Its Applications, (Proc. Symp. Smolenice, 1963) 163-164.

[14] W.C. Shiu, P.C.B. Lam and H.L. Cheng, Supermagic labeling of an s-duplicate of $K_{n, n}$, Congr. Numer. 146 (2000) 119-124.

[15] W.C. Shiu, P.C.B. Lam and S.M. Lee, On a construction of supermagic graphs, J. Combin. Math. Combin. Comput. 42 (2002) 147-160.

[16] B.M. Stewart, Magic graphs, Canad. J. Math. 18 (1966) 1031-1059. https://doi.org/10.4153/CJM-1966-104-7

[17] B.M. Stewart, Supermagic complete graphs, Canad. J. Math. 19 (1967) 427-438. https://doi.org/10.4153/CJM-1967-035-9

Received 21 November 2018

Revised 8 April 2019

Accepted 9 April 2019 